\title{
Postoperative Stevens-Johnson syndrome secondary to piperacillin-tazobactam: A case report
}

\author{
Mihir M Shah, Kelsey Larson, Neilendu Kundu, R Matthew Walsh
}

\section{ABSTRACT}

Introduction: Toxic epidermal necrolysis (TEN) and Stevens-Johnson syndrome (SJS) are severe, potentially fatal cutaneous reactions to medications affecting only 1-2 per million individuals each year. They present as tenderness, erythema and blistering of both the skin and mucus membranes. We describe a case of Stevens-Johnson syndrome linked to piperacillin-tazobactam treatment, which is a previously unreported cause and a rare occurrence in postoperative patients. Case Report: A 37-year-old female with chronic pancreatitis post-total pancreatectomy with auto-islet transplant was re-admitted on postoperative day 12 with abdominal pain and leukocytosis. Her workup revealed a small intraabdominal fluid collection suspicious for an abscess and she was empirically started on piperacillin-tazobactam. She developed hives on her extremities with associated itching after four days of treatment. Piperacillin-tazobactam was discontinued, diphenhydramine was administered for symptomatic relief, and she was discharged on an oral regimen of ciprofloxacin and metronidazole. She returned the following day with a rash extending onto her chest, abdomen, feet, face, lips and mouth, with

Mihir M Shah¹, Kelsey Larson¹, Neilendu Kundu ${ }^{1}$, R Matthew Walsh ${ }^{2}$

Affiliations: ${ }^{1} \mathrm{MD}$, Resident, General Surgery, Cleveland Clinic Foundation, Cleveland, Ohio, USA; $2 \mathrm{MD}$, Faculty, General Surgery, Cleveland Clinic Foundation, Cleveland, Ohio, USA

Corresponding Author: Mihir M Shah, 35 Severance Circle, Apt 311, Cleveland Heights, Ohio, USA, 44118; Ph: (+1) 215-200-6256; Fax: (+1) 216-445-7653; Email: mihir253@yahoo.co.in newly appeared bullae covering approximately 35-50\% of body surface. A skin biopsy was consistent with SJS, with piperacillin-tazobactam identified as the likely causative agent. Conclusion: The most common medications linked to TEN/SJS are sulfonamide antibiotics (especially sulfamethoxazole/trimethoprim), cephalosporins, quinolones, allopurinol, carbamazepine, phenytoin, phenobarbital, and NSAIDs (oxicam-type). While medications the most common cause, mycoplasma pneumoniae and herpes simplex virus (HSV) have also been linked to SJS. The mortality rate of biopsyconfirmed SJS ranges from 1-5\%, whereas the mortality of TEN ranges from $25-35 \%$. Though many patients experience pruritis and/or rashes as side-effects to medications given postoperatively, it is important to keep in mind more serious reactions such as TEN and SJS as these conditions as they have a substantial associated morbidity and even mortality.

Keywords: Stevens-Johnson syndrome, Toxic epidermal necrolysis, Piperacillin-tazobactam

$$
* * * * * * * * *
$$

Shah MM, Larson K, Kundu N, Walsh RM. Postoperative Stevens-Johnson syndrome secondary to piperacillin-tazobactam: A case report. International Journal of Case Reports and Images 2013;4(6):316-320.

$* * * * * * * * *$

doi:10.5348/ijcri-2013-06-321-CR-6

\section{INTRODUCTION}

Stevens-Johnson syndrome (SJS) and toxic epidermal necrolysis (TEN) are rare, but severe, potentially fatal exfoliating dermatitis conditions 
affecting only 1-2 per million individuals annually [1]. They typically present as tenderness, erythema, and blistering of both skin and mucus membranes [1]. We report a case of SJS linked to piperacillin-tazobactam treatment, which is a previously unreported cause and a rare occurrence in postoperative patients.

\section{CASE REPORT}

A 37-year-old female with a past medical history significant for Turner's syndrome, type-II diabetes mellitus and chronic pancreatitis, had undergone pancreatic duct drainage and partial pancreatic head resection (Frey procedure) 18 months prior to this admission. She had persistent pancreatic pain requiring narcotics and repeated hospitalizations. She presented to the clinic with chronic, epigastric abdominal pain. A discussion was held and she elected to proceed with total pancreatectomy with auto-islet transplant, which was subsequently performed at our institution. Her postoperative course was unremarkable and she was discharged on postoperative day (POD) 11 in satisfactory condition.

The patient returned the day after discharge with worsening abdominal pain, mild distention, and worsened leukocytosis. A computed tomography (CT) scan was performed demonstrating a $4 \times 2 \mathrm{~cm}$ fluid collection in the mesentery. She was empirically started on piperacillin-tazobactam $3.37 \mathrm{mg}$ IV Q6 hrs after obtaining samples for blood and urine cultures. Her blood and urine cultures were negative, she remained afebrile, and her white blood cell count continued to decrease. Prior to discharge four days later, she developed hives and itching on her extremities. Piperacillin-tazobactam was stopped and she was administered diphenhydramine for relief of symptoms. Her symptoms were controlled and she was discharged home in stable condition on an oral regimen of ciprofloxacin $250 \mathrm{mg}$ BID and metronidazole $500 \mathrm{mg}$ daily.

On the evening of discharge (POD 16), the patient noted spreading of her rash onto her chest, abdomen, feet, mouth and lips with the appearance of bullae. She developed fever, chills, rigors as well as dysuria and oropharyngeal burning. She returned to the emergency department the next morning. Upon evaluation, she was noted to have $35-50 \%$ of her body surface area covered with a rash and bullous lesions (Figure 1A-D). Initial concern was for TEN versus SJS. Dermatology and ophthalmology were promptly consulted, and a skin biopsy was obtained. IgA levels and blood cultures were negative. Her skin lesions were treated with Vaseline gauze (Covidien Mansfield, MA) and Kerlix (Covidien Mansfield, MA), and she was started on solumedrol 60 mg IV BID.

Skin biopsy sections demonstrated interface dermatitis characterized by detached epidermis with numerous dyskeratotic keratinocytes and scant perivascular infiltrate within the dermis, consistent with SJS (Figure 2A-C). Piperacillin-tazobactam was suspected as the likely causative agent after a thorough review by all teams involved. She did not have any ophthalmologic issues. After stabilization and initial treatment, she was transferred to the regional burn center for continuation of management. She was initially managed with local skin care and IV antibiotics with significant improvement. She was subsequently lost to follow-up after discharge from the burn center. Unfortunately she is now listed as deceased in electronic medical record system, with unknown date and cause of death.
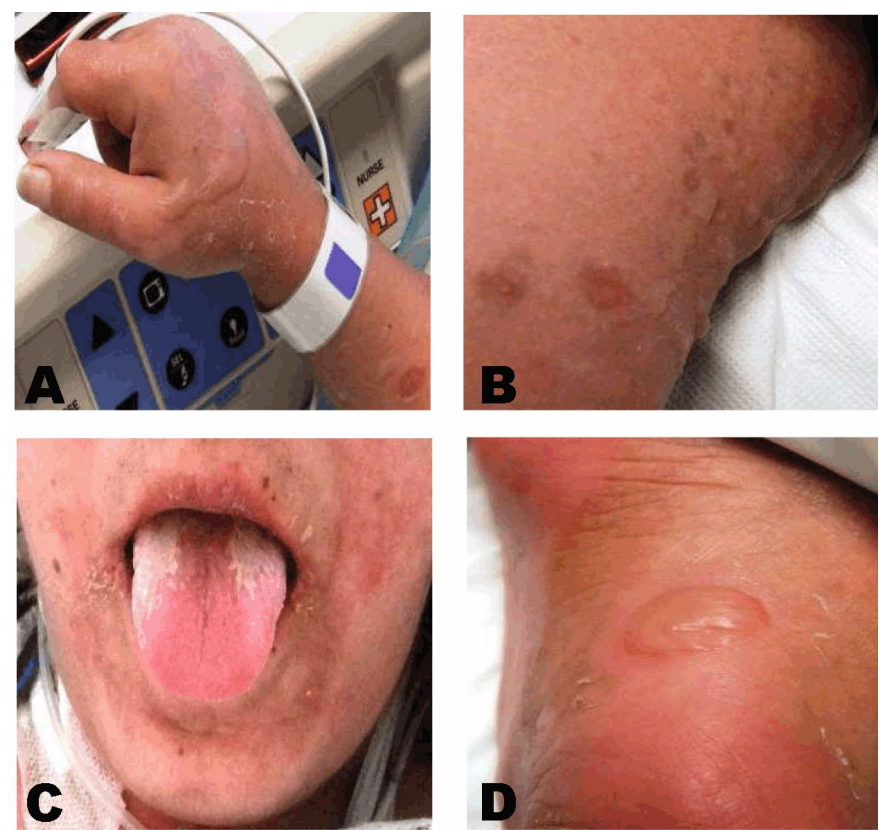

Figure 1: (A) Right hand and forearm with flaccid bullae, superficial desquamation and a solitary denuded bulla, (B) Right medial thigh with tense and flaccid vesicles and bullae upon a background of erythema, (C) Upper cutaneous lip, vermillion border and oral aperatures with superficial desquamation. Tongue without vesicles, bullae or desquamation, (D) Right anterior thigh with solitary serousfilled bulla within a field of superficial desquamation.

\section{DISCUSSION}

Epidemiology: TEN and SJS occur in approximately $1-2$ per million individuals each year, with relatively similar rates reported in multiple countries around the world $[1,2]$. Small differences in the rates of SJS and TEN can likely be attributed to genetics, medication availability, prescribing practices and other concurrent conditions, including cancers and infection. One notable population with a significantly increased risk for SJS and TEN includes those infected with HIV, as the annual incidence is 1000 times higher than the general population (1/1000) [1,3,4].

Clinical Evaluation: Initial presentation of the disease typically begins several days prior to cutaneous manifestations. Symptoms include fevers, keratitis sicca, odynophagia and malaise making it difficult to differentiate from routine drug reactions $[1,2,5]$. When skin lesions first appear, they often begin on the trunk 


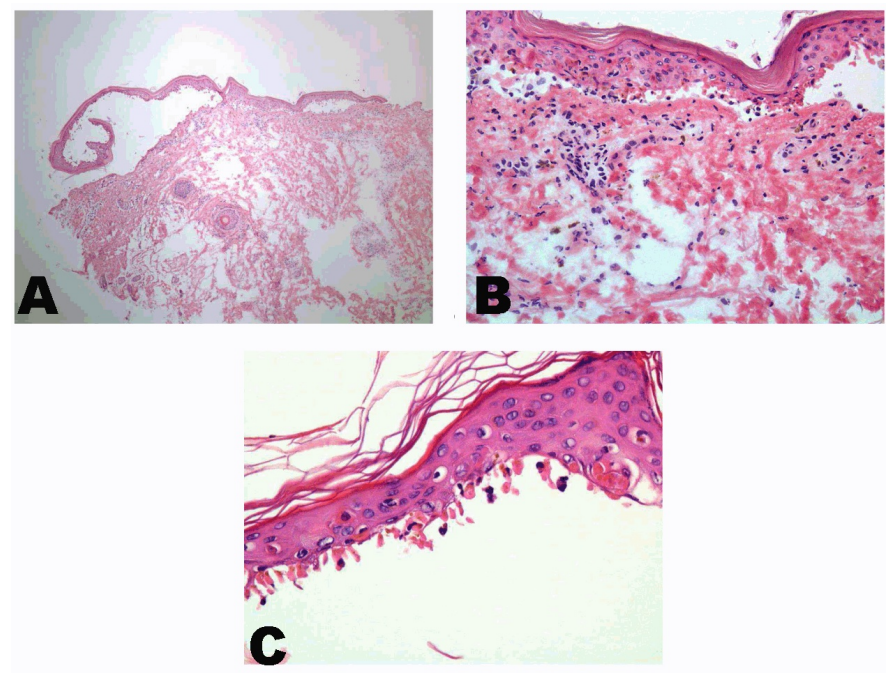

Figure 2: (A) Low power image demonstrating subepidermal blister, (B) Within the dermis there is a sparse perivascular lymphocytic infiltrate with interface change and numerous dyskeratotic cells, (C) High power image of the epidermis highlighting the numerous dyskeratotic keratinocytes.

and face in addition to the palms and soles and appear as dusky red, flat, poorly delineated areas. They then continue to spread outward $[1,5]$. Evidence of involvement of the buccal, genital, and/or ocular mucosa should raise suspicion of SJS or TEN and occurs in $>90 \%$ of patients [1]. Ocular involvement early is common, and can include conjunctivitis, edema, erythema, crusting, discharge erosions and corneal ulcerations $[1,6,7]$.

Epidermal detachment usually occurs as a slightly later secondary manifestation. The patent can be tested by applying lateral mechanical pressure and observing for detachment (Nikolsky's sign) or visualizing bullae extending to surrounding skin when vertical pressure is applied (Asbor-Hansen sign). While not pathognomonic for SJS or TEN, these exam findings are helpful for ascertaining the danger of the reactions. In addition to epidermal involvement, the respiratory, gastrointestinal (including the liver), and urinary systems can be involved [8-10].

The TEN survivors have a 50\% risk of long-term sequelae including changes in pigmentation of the skin and ocular complications ranging from simple (keratitis sicca, 46\%) to severe (visual loss 5\%, corneal ulceration $2 \%)[1,11]$.

Etiology: The pathophysiology of SJS and TEN remains unclear. Current theories support immunemediated phenomena, resulting in keratinocyte apoptosis followed by necrosis. The generalized mechanism for this reaction is $\mathrm{MHC}-1$ drug presentation leading to clonal expansion of cytotic T-cells, found in high numbers in the blisters of SJS/TEN patients [1]. However, additional mediators including FasL and granulysin (cytotoxic molecule), are likely to play primary roles in the actual generalized apoptosis of the keratinocytes $[1,5]$. The Fas-FasL pathway has been linked to apoptosis in SJS/TEN via triggered intracellular DNA degradation [5]. The use of IVIG as treatment for SJS/TEN is based on this link between the disease and FasL $[1,5]$. The exact pathway by which a medication leads to secretion or activation of Fas-FasL and/or ganulysin and thus keratinocyte apoptosis remains to be explained.

Genetics: The link between SJS and genetic HLA class was first reported in the Han Chinese population with a strong association between HLA-B*1502 and carbamazepine $[1,12]$. This association was confirmed in other Asian populations. However, this was not valid in European populations, leading to the conclusion that genetic markers are not specific in predicting SJS/TEN susceptibility in the general population [1].

Drugs: Numerous medications have been identified as causative agents for SJS and TEN. The most common medications linked to SJS/TEN over short-term use include trimethoprim-sulfamethoxazole and other sulfonamides, aminopenillins, cephalosporins, quinolones, and chlormezaone. Some medications are linked to SJS/TEN following long-term use, such as carbamazepine, phenytoin, phenobarbital, valproic acid, NSAIDs (oxicam-type), nevirapine, lamotrigine, sertraline, and allopurinol. With these medications, SJS/TEN usually occurs within two months after beginning treatment $[1,3]$.

Disease: While medications, the primary cause of SJS and TEN, mycoplasma pneumoniae and HSV have also been linked to development of these diseases without medication exposure [1]. HIV patients are at significantly higher risk for development of SJS/TEN compared to the general population.

Diagnosis: The diagnosis of SJS versus TEN relies on clinical vigilance and pathologic confirmation. SJS is defined by mainly isolated lesions with $<10 \%$ body surface area skin detachment, while TEN is a more severe and wide-spread form with widely confluent lesions and skin detachment covering $>30 \%$ of the body surface area [1]. Skin biopsy is the only way to confirm definitive diagnosis and rule-out other autoimmune blistering diseases, fixed drug reactions, and staphylococcal scaled skin syndrome. Histopathologic analysis reveals necrosis of the epidermis involving all layers, with evidence of keratinocyte apoptosis. Direct immunofluorescent staining is also carried out to ruleout autoimmune causes [1].

Given the severe nature of SJS/TEN and its initial presentation, a rapid diagnostic test to predict SJS versus benign drug reaction is being developed [13] Patients who are diagnosed with SJS have increased systemic levels of FasL as well as increased granulysin levels. While the FasL serum levels are too low to measure, the granulysin serum levels can be measured in rapid fashion to yield positive versus negative results in 15 minutes, similar to a rapid strep test. This method had sensitivity of $80 \%$ and specificity of $95.8 \%$ for SJS/TEN versus benign drug reaction. While not widely available for clinical use, further development of fast, efficient, early diagnostic tools is important for improved patient outcomes in the future. 
Management: Initial management when a diagnosis of SJS/TEN is suspected begins with immediate cessation of all new medications as well as urgent consultation to dermatology and ophthalmology. Universally accepted treatment includes monitoring volume status, evidence of sepsis, and conservative wound treatment without debridement [1]. The role of IVIG in treatment in addition to corticosteroids is generally accepted, although controversy remains regarding the exact details of the treatment [14].

Prognosis: The SCORTEN scoring system was developed as a validated means for predicting disease severity and mortality associated with SJS/TEN (Table 1 and Table 2) [1]. This score is useful for determination of appropriate treatment (all scores $>3$ should be treated in ICU and transferred to a regional burn center as soon as possible) and patient/family counseling. The leading cause of death in most patients is sepsis leading to acute renal failure [1]. The patient in this case report had a SCORTEN score of 4 (tachycardia $+>10 \%$ body area involvement + glucose elevation + bicarbonate $>20$ ), corresponding to a $58.3 \%$ mortality.

Table 1: SCORTEN Scoring System Each "yes" answer to the parameter yields one point, each "no" answer is worth zero points.

Age $>40$ years old?

Presence of malignancy?

Tachycardia ( $>120$ beats per minute)?

Initial surface of epidermal detachment $>10 \%$ ?

Serum urea $>10 \mathrm{mmol} / \mathrm{L}$ ?

Serum glucose $>14 \mathrm{mmol} / \mathrm{L}$ ?

Bicarbonate $>20 \mathrm{mmol} / \mathrm{L}$ ?

Table 2: The sum of the SCORTEN criteria yields a score which is correlated with a predicted mortality.

\begin{tabular}{cc} 
SCORTEN (sum of 1A items) & Predicted \% mortality \\
\hline $0-1$ & $3.2 \%$ \\
2 & $12.1 \%$ \\
3 & $35.8 \%$ \\
4 & $58.3 \%$ \\
$>5$ & $90 \%$
\end{tabular}

\section{CONCLUSION}

While many patients experience pruritis and/or rashes as side-effects to medications given postoperatively, it is important to keep in mind more serious reactions such as toxic epidermal necrolysis and Stevens-Johnson syndrome as these conditions can be rare causes of post-operative morbidity and mortality. If there is any concern for possible toxic epidermal necrolysis or Stevens-Johnson syndrome, prompt consultations to dermatology and ophthalmology should be obtained, possible causative agents should be immediately discontinued, and early transfer to a burn center should be initiated following confirmed diagnosis.

$$
* * * * * * * * *
$$

\section{Author Contributions}

Mihir Shah - Conception and design, Acquisition of data, Analysis and interpretation of data, Drafting the article, Critical revision of the article, Final approval of the version to be published

Kelsey Larson - Conception and design, Acquisition of data, Analysis and interpretation of data, Drafting the article, Critical revision of the article, Final approval of the version to be published

Neilendu Kundu - Acquisition of data, Analysis and interpretation of data, Drafting the article, Critical revision of the article, Final approval of the version to be published

R Matthew Walsh - Conception and design, Acquisition of data, Analysis and interpretation of data, Drafting the article, Critical revision of the article, Final approval of the version to be published

\section{Guarantor}

The corresponding author is the guarantor of submission.

\section{Conflict of Interest}

Authors declare no conflict of interest.

\section{Copyright}

(C) Mihir Shah et al. 2013; This article is distributed under the terms of Creative Commons Attribution 3.0 License which permits unrestricted use, distribution and reproduction in any means provided the original authors and original publisher are properly credited. (Please see www.ijcasereportsandimages.com/copyright-policy.php for more information.)

\section{REFERENCES}

1. Harr T, French LE. Toxic epidermal necrolysis and Stevens-Johnson Syndrome. Orphanet Journal of Rare Diseases 2010;5:39.

2. Sanmarkan AD, Sori T, Thappa DM, Jaisankar TJ. Retrospective analysis of Stevens-Johnson Syndrome and Toxic Epidermal Necrolysis over a period of 10 years. Indian Journal of Dermatology 2011 Jan-Feb;56(1):25-9.

3. Roujeau JC, Kelly JP, Naldi L, et al. Medication use and the risk of Stevens-Johnson syndrome or toxic epidermal necrolysis. N Engl J Med 1995;333(24):1600-7.

4. Saiag P, Caumes E, Chosidow O, Revuz J, Roujeau JC. Drug-induced toxic epidermal necrolysis (Lyell syndrome) in patients infected with the human 
immunodeficiency virus. Journal of the American Academy of Dermatology 1992;26(4):567-74.

5. Chung WH, Hung SI. Genetic Markers and Danger Signals in Stevens-Johnson Syndrome and Toxic Epidermal Necrolysis. Allergology International 2010;59(4):325-32.

6. Chang YS, Huang FC, Tsent SH, Hsu CK, Ho CL, Sheu MH. Erythema multiforme, Stevens Johnson Syndrome, and Toxic Epidermal Necrolysis: Acute ocular manifestations, causes and management. Cornea 2007;26(2):123-9.

7. Sotozono C, Ueta M, Koizumi N, et al. Diagnosis and treatment of Stevens-Johnson Syndrome and Toxic Epidermal Necrolysis with ocular complications. Opthamology 2009;116(4):685-90.

8. Lebargy F, Wolkenstein P, Gisselbrecht $\mathrm{M}$, et al. Pulmonary Complications in toxic epidermal necrolysis: A prospective clinical study. Intensive Care Medicine 1997;23(12):1237-44.

9. Sugimoto $\mathrm{Y}$, Mizutani H, Sato T, Kawamura N, Ohkouchi K, Shimizu M. Toxic epidermal necrolysis with severe gastrointestinal mucosal cell death: a patient who excreted long tubes of dead intestinal epithelium. Journal of Dermatology 1998;25(8):533-8.
10. Ducic I, Shalom A, Rising W, Nagamoto K, Munster AM. Outcome of patients with toxic epidermal necrolysis syndrome revisited. Plastic and Reconstructive Surgery 2002;110(3):768-3.

11. Yip LW, Thong BY, Lim J, et al. Ocular manifestations and complications of Stevens Johnson Syndrome and Toxic Epidermal Necrolysis: an Asian series. Allergy 2007;62(5):527-31.

12. Chung WH, Hung SI, Hong HS, et al. Medical Genetics: a marker for Stevens-Johnson Syndrome. Nature 2004;428(6982):486.

13. Fujita Y, Yoshioka N, Abe R, et al. Rapid immunochromatographic test for serum granulysin is useful for the prediction of Stevens Johnson Syndrome and Toxic Epidermal Necrolysis. Journal of the American Academy of Dermatology 2011 July;61(1):65-8.

14. Worswick S, Cotliar J. Stevens-Johnson Syndrome and Toxic Epidermal Necrolysis: a review of treatment options. Dermatologic Therapy 2011 MarApril;24(2):207-18.
Access full text article on other devices

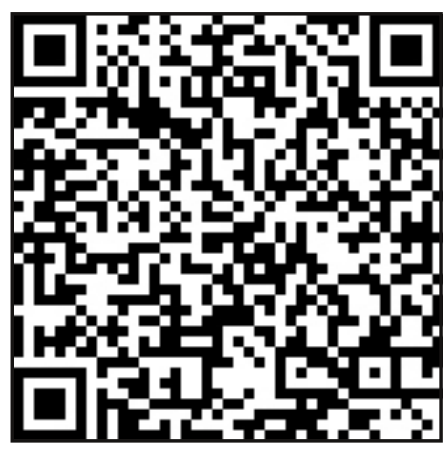

Access PDF of article on other devices

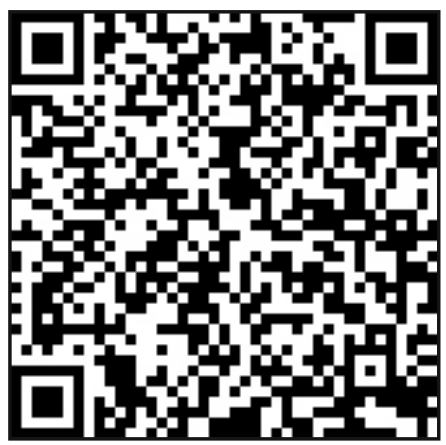

